\title{
Ni educación, ni deferencia ciega. Hacia un modelo crítico para la valoración de la prueba pericial
}

\author{
Neither Education, nor Blind Deference: Towards a \\ Critical Model for the Assessment of Expert Evidence
}

Rachel Herdy ${ }^{*}$

Recepción: 19/12/2019

Evaluación: 15/1/2020

Aceptación final: 17/2/2020

\begin{abstract}
Resumen: Este artículo discute el argumento de Carmen Vázquez a favor de un modelo educacional para la valoración de la prueba pericial. No se enfoca en las herramientas institucionales que Vázquez cree podrían promover la educación de decisores, sino en los presupuestos psicológicos y epistémicos de su trabajo. Por un lado, cuestiona su suposición empírica de que decisores son cognitivamente capaces de comprender el razonamiento de los expertos; por otro, argumenta que la afirmación de Vázquez de que la deferencia es una actitud irracional que amenaza la legitimidad de las decisiones judiciales parece ignorar el papel que el razonamiento basado en la autoridad generalmente juega en la justificación judicial. El artículo esboza un modelo para la valoración de la prueba pericial que no promueve ni la educación, ni la deferencia ciega. En cambio, propone un modelo crítico y más democrático en el que no solo el juez o jurado científicamente educado tiene la capacidad de llegar a una decisión justificada sobre la base de la información transmitida por los expertos, sino la sociedad como un todo.
\end{abstract} Palabras clave: prueba pericial, educación, deferencia, razones de autoridad.

Doctora en Sociología. Profesora de Teoría del Derecho de la Universidade Federal do Rio de Janeiro (UFRJ), Río de Janeiro, Brasil. Correo electrónico: rachelherdy@direito.ufrj.br. 
Rachel Herdy

\begin{abstract}
This paper discusses Carmen Vázquez's argument in favor of an educational model for the assessment of expert evidence. It focuses not on the institutional arrangements that Vázquez believes might promote the education of decision-makers, but on the psychological and epistemic presuppositions of her work. One the one hand, it questions her empirical assumption that decision-makers are cognitively capable of understanding the reasoning of experts; on the other, it argues that Vazquez's claim that deference is an irrational attitude that threatens the legitimacy of judicial decisions seems to disregard the role that authoritative reasoning usually plays in judicial justification. The paper sketches a model for the assessment of expert evidence that supports neither education, nor blind deference. Instead, it proposes a critical, more democratic model in which not only the scientifically educated judge or jury is capable of reaching a justified decision on the basis of expert testimony - but society as a whole.
\end{abstract}

Keywords: Expert evidence, education, deference, authoritative reasons.

Si deseamos que los jueces tomen decisiones racionales respecto de los hechos que dependen de la prueba pericial, es necesario que ellos comprendan las inferencias realizadas por el experto - este es el argumento central del artículo de Carmen Vázquez, "El diseño normativo de las pruebas periciales, a propósito del razonamiento inferencial de los expertos y la comprensión judicial"- - Después de considerar brevemente el supuesto psicológico de su argumento (i. e., la capacidad de los jueces), Vázquez explora una serie de herramientas procesales que entiende oportunas y adecuadas para ayudar al juez a educarse. El artículo termina con una discusión sobre los cuidados que debemos tener con las actividades anteriores a la conformación del conjunto de informaciones probatorias, es decir, con lo que ocurre en el llamado momento de descubrimiento. Para la autora, la originalidad de su propuesta radicaría en el empleo conjunto de tales herramientas en un mismo ordenamiento jurídico, y en el énfasis que se presta al tiempo de la práctica de la prueba pericial.

A partir del artículo de Vázquez, la pregunta que me parece importante plantear es la siguiente: cuando los expertos presentan informaciones (evidencias, generalizaciones, interpretaciones, inferencias) basadas en 
conocimientos de sus respectivas áreas, ¿qué esperanza podemos tener de que jueces legos puedan evaluar racionalmente la información aportada? Luego se ve que mi punto de discordancia con la autora se localiza no tanto en sus propuestas concretas de un modelo educacional de la prueba pericial, sino más propiamente en los presupuestos de su argumentación. Las herramientas sugeridas — como la oralidad y contradicción en la práctica de la prueba pericial; la convocación de asesores externos que actuarían de manera análoga a los law clerks; la posibilidad de peritos terceros para revisar las pruebas cuando existe un desacuerdo importante entre los peritos de las partes o entre ellos y los oficiales; e incluso la idea de un hot-tub o junta de peritos donde se reunirían los peritos, las partes y los juzgadores para un debate en estilo académico- parecen promisoras y útiles incluso para el modelo deferencial crítico cuyas líneas generales presentaré en la sección final de esta discusión.

Así es que la reflexión que propongo se centra en los presupuestos de las propuestas de Vázquez. Esos presupuestos son dos: el primero - explicitado por la autora- es de carácter empírico-psicológico, y se refiere a las capacidades cognitivas del juez y sus habilidades para la adquisición de conocimientos expertos; el segundo - que sigue implícito en sus consideraciones- es de carácter teórico-epistémico, y se refiere a la naturaleza de las razones que justifican las decisiones judiciales. Esos dos presupuestos están relacionados: la justificación de la decisión sobre los hechos que demandan conocimientos especializados depende de la comprensión, por parte de juzgadores cognitivamente capaces, de las razones de primer orden que posee el experto.

Propongo estructurar esta discusión de la siguiente manera. En la primera sección, antes de presentar los argumentos contra los supuestos de Vázquez, recuperaré brevemente los antecedentes teóricos del modelo educacional. En la segunda sección, presentaré el primer punto central de mi discusión: voy a argumentar que no hay buenas razones para presuponer que los jueces tienen la disposición o son capaces de comprender las inferencias de los expertos. A continuación, presentaré el segundo punto importante de mi argumento: la deferencia a razones de autoridad no solo es racional, sino que constituye un fenómeno característico de la justificación judicial. En este momento, esbozaré un modelo crítico y más demo- 
crático para el tratamiento de la prueba pericial. La discusión termina con una conclusión que retoma los puntos principales y sitúa el problema de la valoración racional de las pruebas periciales en un contexto más amplio.

\section{Los antecedentes del modelo educacional}

La discusión planteada por Vázquez remite a un artículo célebre de Joseph Miller y Ronald Allen publicado en 1993, el mismo año en que la Corte Suprema de los Estados Unidos decidió por primera vez sobre los criterios de admisibilidad del testimonio experto (caso Daubert) (Miller y Allen, 1993). ${ }^{1}$ Aunque la autora no haga referencia directa a las ideas de Miller y Allen en su artículo, la influencia de la apuesta educacional de los juristas norteamericanos, principalmente del último, se revela en sus trabajos anteriores sobre la prueba pericial y científica (Vázquez, 2015). Por lo tanto, para poder acercarme mejor a los presupuestos que están en juego detrás de un diseño institucional direccionado a la educación de los jueces, dedicaré esta parte inicial de mi discusión a los argumentos de Allen. La elucidación y consideración crítica de sus argumentos pueden contribuir para comprender mejor el contexto en que se sitúa la propuesta educacional de Vázquez.

Como dije, el artículo de Miller y Allen fue publicado en el mismo año de la decisión del caso Daubert. ${ }^{2}$ De este caso paradigmático, resultó un desplazamiento de la responsabilidad de las comunidades de expertos hacia los jueces. Muy resumidamente, el caso Daubert consistió en una decisión sobre la validez del test Frye, establecido en un caso de 1923, luego de la promulgación de las Federal Rules of Evidence en 1975 (en adelante FRE). El test Frye era utilizado - y todavía lo es, en algunos tribunales estaduales - para determinar la admisibilidad de los testimonios expertos. Su aplicación consistía en verificar si la teoría ofrecida por el experto

1 El artículo de Miller y Allen produjo una serie de discusiones entre los estudiosos del derecho probatorio norteamericano, y anticipó la discusión que se iniciaría en el mismo año con Daubert. Véase, en el mismo volumen, Epstein (1993).

2 Es curioso notar que hay una sola referencia a la decisión de la Suprema Corte en el artículo de Miller y Allen, la cual aparece en una nota de pie de la página 1141. La nota utiliza un símbolo diferente, dando la impresión de haber sido incluida posteriormente a la redac- 
estaba "suficientemente establecida para ser generalmente aceptada en la comunidad jurídica relevante". La discusión concreta del caso era sobre el uso del polígrafo para absolver a un imputado que anteriormente había confesado el crimen. La Corte Suprema decidió que el test Frye había sido superado por las FRE, y en una decisión confusa (y sobre la cual mucho se ha escrito) indicó cuatro factores que pueden ser considerados para decidir respecto de la pertinencia de la prueba ofrecida por el experto - y la cuestión de la pertinencia de esta prueba, advirtió la Corte, es un problema de fiabilidad, que, a su vez, se mide en términos de validez científica-. Los cuatro factores son: si la teoría o método puede ser y efectivamente fue testeado; si la teoría o método fue publicado o de alguna forma revisado por pares; la tasa de error conocida o potencial del método; y la aceptación general de la teoría en la comunidad científica relevante (una repetición del test Frye). Una secuencia de casos fue analizada por la Corte en la misma década - que componen la "trilogía Daubert" - y otras indicaciones fueran agregadas: que los estudios no hayan sido preparados para ser presentados en el litigio; que los jueces pueden examinar también la conclusión; que la decisión del juez de admitir o no la prueba es discrecional; y que el test Daubert se aplica también a las pruebas técnicas. ${ }^{3}$ Si antes de Daubert la admisibilidad del testimonio experto dependía de una deferencia a la comunidad de expertos, a partir de 1993 se exige la adopción de un modelo de educación (defendido por Miller y Allen meses antes de la decisión) para que el juez sea capaz de individualmente valorar la fiabilidad de la prueba ofrecida por el experto.

Los argumentos presentados por Miller y Allen en 1993 fueron reforzados en un artículo que Allen publicó solo en 2013, dos décadas después, bajo el título "El desafío conceptual de la prueba pericial". Cito uno de los pasajes más inquietantes de Allen, que aparece en los dos textos:

¿Hay casos que presentan problemas de decisión que desafíen la capacidad de los investigadores de hechos para comprenderlos?

ción del artículo. En la nota, los autores señalan que Daubert "destaca la distinción entre deferencia y educación".

3 Para un listado completo y crítico, véase Haack (2019). 
Quizás la respuesta es no. Los déficits de los investigadores de hechos jurídicos no parecen ser cognitivos, sino informativos. Jueces y jurados carecen de conocimiento sobre muchas cosas, como la ciencia y la tecnología, pero no hay ninguna razón para que no puedan dominar adecuadamente los campos relevantes (Allen, 2013, p. 51, la traducción es mía).

Para Allen, hay solo dos posibles soluciones al problema de tomar una decisión racional sobre el valor de la información que los expertos aportan a los tribunales: o bien a los jueces y miembros del jurado le ofrecen la información básica que necesitan para comprender lo que dice el experto, o bien los juzgadores tendrán que deferir irracionalmente al juicio de otra persona. "Deferir" significa adoptar el punto de vista de otra persona como correcto, pero no porque se entiende y se está de acuerdo, sino porque se delega esa decisión en otra persona. Deferir difiere de "educar", que significa entender el proceso de razonamiento que condujo el experto a su conclusión. La "vulnerabilidad epistémica" del juzgador — una expresión de Allen- podría ser corregida solamente por la educación. Es necesario que "el tribunal pueda concluir independientemente que la opinión del experto está realmente fundada en conocimiento" (Allen, 2013, p. 62).

Allen explica que la deferencia y la educación no son conceptos analíticamente distintos, sino puntos opuestos en el mismo espectro. También reconoce que la deferencia es una variable omnipresente de la decisión judicial, ya que es un fenómeno que se manifiesta, aunque en menor medida, cuando valoramos cualquier testimonio. Sin embargo, no explora la posibilidad de un camino intermedio, rechazando por completo la deferencia, porque entiende que esta actitud es contraria a la racionalidad; y la racionalidad es una de las "aspiraciones fundamentales" de los sistemas jurídicos de los Estados liberales (Allen, 2013, p. 43). Allen pondera que el objetivo no es comprender completamente un campo, sino "lo suficiente para que haya una deliberación racional” (Allen, 2013, p. 51). Pero ¿cómo sería posible saber qué parte del conocimiento es suficiente si ni siquiera puedo entender el vocabulario técnico empleado, los supuestos fácticos de fondo o incluso lo que puede ser relevante? Dice también que los órganos de toma de decisiones colectivas, como los tribunales de jueces o jurados, 
a menudo son superiores a los jueces individuales, porque sería suficiente que el grupo en su conjunto domine el campo del experto. Eso parece tener sentido, pero es un problema para los sistemas de justicia de la tradición continental, donde las decisiones sobre los hechos frecuentemente ocurren ante un juez singular. Finalmente, reconoce que áreas más difíciles existen, como la "física cuántica"; sin embargo, sostiene que, sobre estos temas, no se litiga. Sobre este punto, me encuentro pensando frecuentemente en el uso cada vez mayor del concepto de "cuántica" para calificar áreas esotéricas como astrología cuántica y coaching cuántico, y sobre la posibilidad de que alguien algún día cuestione judicialmente el uso de tal expresión para engañar pacientes y consumidores.

El único argumento que Allen supone que podría usarse en contra de un modelo educacional es económico: los costos. Pero para él, los costos con la educación están justificados, porque ya hay procesos que presentan costos altos sin requerir expertos: procesos que incluyen muchos testigos o complejas cadenas de inferencias probatorias. Para él, este tipo de discusión sobre costos revela la naturaleza "bizarra" o "extravagante" de la controversia (Allen, 2013, p. 52). A mí me parece que extravagante es pensar que esta sea una discusión extravagante, especialmente a la luz de desafíos político-económicos muy serios: faltan recursos a los Estados; vivimos un período de estratificación de áreas de especialización; trabajamos con un contexto de ampliación del acceso a la justicia; y discutimos la posibilidad de utilizar la inteligencia artificial para la toma de decisiones judiciales en el mediano plazo.

La propuesta de Allen se tomó rápidamente en serio. En 1997, pocos años después de la decisión de la Corte Suprema, Edward Imwinkelried - el teórico del razonamiento probatorio más citado en Estados Unidos según "the Leiter Report" de 2007- propuso, como corolarios procesales del modelo educacional, un conjunto de mecanismos didácticos para que jueces y jurados pudieran ser educados en el área de especialización del experto (Imwinkelried, 1997). Estos mecanismos complementarían las pruebas presentadas por testigos expertos, pero no los sustituirían. La solución de Imwinkelried fue transformar el tribunal en una especie de "salón de clases" en que los jueces deberían asumir el rol de maestros - deberían comprender la información experta e informar a los jurados sobre la ciencia en cuestión-; los expertos neutrales designados por el tribunal actua- 
rían como monitores o asistentes de enseñanza — ayudarían a los jurados a asegurarse de que entenderían la explicación del juez-; y los jurados, a su vez, serían los estudiantes - harían preguntas a los monitores expertos en un ambiente de total libertad para el diálogo y el aprendizaje-. Lo que Imwinkelried no aclara es cómo el juez lograría el conocimiento suficiente para asumir esta función didáctica.

La propuesta de Vázquez que aquí se discute se suma a este grupo de académicos del derecho probatorio norteamericano que apuestan al modelo educacional para el tratamiento de la prueba pericial. Vázquez y Allen comparten el pensamiento de que el elemento esencial para que una decisión judicial basada en el conocimiento experto esté justificada es la comprensión, por parte del juzgador, del proceso de razonamiento del perito ${ }^{4}$. Según la autora, los jueces deben comprender tres elementos, presentes en el razonamiento del experto: "los hechos relevantes" del caso; las generalizaciones "independientes de los hechos del caso"; y las relaciones entre ellos.

Esta distinción tripartita de los elementos que deben ser comprendidos por el juzgador puede ilustrarse con la ayuda de la distinción que David Faigman propone entre pruebas diagnósticas, pruebas estructurales e inferencia G2i, respectivamente (Faigman, 2014). Las pruebas diagnósticas ayudan al juzgador a comprender los hechos relevantes de naturaleza individual o particular, también conocidos como "hechos adjudicativos". Las generalizaciones independientes, a su vez, dependen de pruebas estructurales, las que se refieren a hechos o proposiciones generales sobre el mundo empírico que trascienden (y son relevantes) para la solución del caso. Por ejemplo, para saber si los daños causados a la víctima V (prueba diagnóstica) resultaron del consumo de la droga $\mathrm{D}$ será necesario que el experto afirme que, en general, con base en los estudios científicos $\mathrm{X}, \mathrm{Y}$ y Z, la droga D causa los daños sufridos por V (prueba estructural). ${ }^{5}$ Sin embargo, como bien señala Vázquez, para que el juzgador sea capaz de comprender las inferencias del perito, además de acceder a las pruebas diagnósticas y estructurales, es necesario que sea capaz de relacionarlas

5 Las pruebas estructurales pueden ingresar en el proceso también como alegaciones de los propios jueces - en cualquier instancia- relacionadas con hechos legislativos. Dichas ale- 
con los hechos relevantes del caso - lo que Faigman llama de inferencia "G2i" (de lo general a lo individual) -. En la práctica, podemos pensar en expertos que se pronuncian sobre estas dimensiones probatorias por separado o en conjunto. En cualquier caso, para que el juez sea efectivamente capaz de comprender y aprovechar la inferencia del experto, y así tomar una decisión racional sobre los hechos de un caso —-sostiene Vázquez-es necesario que entienda los tres elementos anteriores. ${ }^{6}$

En el campo adversario (i. e., del modelo deferencial), unos de los trabajos más antiguos y citados es un estudio de 1901 realizado por el juez estadounidense Learned Hand. Es famosa la crítica de Hand de que el sistema estadounidense hace que "el jurado decida cuando los médicos no están de acuerdo". Para Hand, la figura del experto en el sistema de justicia estadounidense era una verdadera "anomalía": confundían más de lo que ayudaban a los tomadores de decisión. Hand sugiere que la solución sería instituir un consejo de expertos al que se transferiría el poder de decidir sobre los hechos en cuestión. Pero quedamos sin saber cómo el juez garantizaría una selección adecuada de los expertos, que ellos no tengan relaciones con la causa e incluso que no van a discordar. Además, Hand obviamente no podría prever la inmensa estratificación de las áreas de conocimiento experto.

La idea en sí no es mala, y efectivamente ya tenemos experiencias con órganos de decisión jurídica constituidos por jueces con formación en derecho y en ciencias (e.g., los Tribunales Ambientales en Chile y el Tribunal Marítimo en Brasil). El jurista australiano Gary Edmond sugiere algo similar:

gaciones son hechas cuando los jueces deciden fundamentar sus elecciones normativas en proposiciones empíricas de diversas áreas del conocimiento: ciencias sociales, psicología, historia, etc. Cuando las evidencias estructurales son ofrecidas en referencia a alegaciones de hecho legislativo, soportan empíricamente la interpretación normativa del juez. A la luz de la estructura silogística de la decisión judicial, se relacionan, por lo tanto, con su premisa mayor. Sin embargo, en el caso anterior, la prueba estructural aparece como una de las premisas de inferencia probatoria realizada por el experto (y que debe ser comprendida por el juez, según Vázquez). La conclusión de esta inferencia probatoria constituirá la premisa menor del silogismo judicial.

6 Si tomáramos en serio la educación de los jueces, diría que sería necesario más que esto. Además de la comprensión de los tres elementos anteriores, el juzgador bien-educado debería ser capaz también de resolver eventuales conflictos de inferencias entre los peritos. 
propone la creación de un Panel Consultivo Multidisciplinar (Multidisciplinary Advisory Panel - MAP) para ayudar a los jueces en sus decisiones de admisibilidad (Edmond, 2012). El MAP sería constituido por expertos independientes y de variadas áreas que evaluarían de modo pragmático —de forma expedita y estricta - la fiabilidad de las pruebas científicas o técnicas. El MAP publicaría su parecer utilizando pocas referencias, lo que presupone "un grado de deferencia de las cortes". Para Edmond, la propuesta difiere de las demás porque, entre otras cosas, no enfatiza la educación:

El uso de un MAP facilita el compromiso con expertos independientes sin la necesidad de una reforma institucional radical. Tiene el beneficio institucional de eludir parcialmente la necesidad de una alfabetización judicial científica y técnica (Edmond, 2011, la traducción es mía).

Creo que las consideraciones anteriores son suficientes no solo para contextualizar y situar mejor la propuesta de Vázquez, ${ }^{7}$ sino también para esclarecer las dos posiciones que están en conflicto - deferencial y educacional- en lo que concierne a la valoración de la prueba pericial. No obstante, entre ellas hay un punto de consenso: la valoración de las pruebas periciales por parte de los juzgadores legos es un problema que merece atención, y las respectivas reformas que se promuevan en nuestro diseño probatorio no pueden fundarse en suposiciones sin fundamento empírico — como bien señaló Vázquez- ${ }^{8}$ En la siguiente sección, consideraré algunos estudios producidos recientemente que nos llevan a reflexionar

7 Otros trabajos representativos de una propuesta educacional para el tratamiento de la prueba pericial, de las tradiciones del common law y del derecho continental: Dwyer (2008), Gascón (2016).

8 Esta es una preocupación planteada por los estudiosos norteamericanos por lo menos desde 1981, cuando Richard Lempert reclamó la necesidad de que la Suprema Corte considerara los estudios científico-sociales para juzgar la restricción a la $7^{\mathrm{a}}$ Enmienda (el derecho a un juzgamiento por jurados) en casos complejos. Para Lempert, la cuestión si el juzgamiento por jurados en casos que involucraban pruebas complejas (periciales o muy numerosas) violaba el derecho a un juicio justo y racional garantizado por la $5^{\mathrm{a}}$ Enmienda tenía fundamentos empíricos para los cuales todavía no existían evidencias (Lempert, 1981). Véase la continuación de la discusión en Lempert (1992). 
sobre el presupuesto empírico-psicológico de que los jueces son capaces de comprender el razonamiento de los expertos. Tales estudios me ayudarán a apoyar algunos puntos de la presente discusión.

\section{El presupuesto de carácter empírico-psicológico}

Según Vázquez, los estudios empíricos realizados con los juzgadores en materia de prueba pericial "contrastan con la asunción de una incapacidad cognitiva”. La autora no cita directamente los estudios mencionados, dejando a los lectores curiosos por saber más respecto de las conclusiones de tales investigaciones. En cambio, Vázquez hace referencia indirecta a un listado de trabajos sobre el tema que aparece en un artículo de Frederick Schauer y Barbara Spellman (2013).

En efecto, el objetivo de Schauer y Spellman en el artículo aludido es llamar la atención hacia una distinción problemática entre pruebas periciales y testimoniales - por lo tanto, el título “¿Es la prueba pericial realmente distinta?"- Es importante resaltar la naturaleza comparativa del argumento que formulan, y su foco en los jurados como tomadores de decisión. Según Schauer y Spellman, existe un escepticismo injustificado en el diseño procesal norteamericano en relación con la capacidad de los jurados para evaluar las pruebas ofrecidas por expertos. La idea de que el juez debe actuar como un portero (gatekeeper) de informaciones y la existencia de criterios de admisibilidad de pruebas comparativamente más severos en relación con la prueba pericial está fundada en la suposición de que los jurados tienden a sobrevalorar las informaciones ofrecidas por expertos. Este miedo en relación con la capacidad cognitiva de los jurados estaría mal fundado empíricamente, pues los datos muestran que los jurados no sobrevaloran tanto en comparación con los errores de sobrevaloración que ellos también cometen con base en pruebas testimoniales. En las palabras de los autores, hay una sobrevaloración de la sobrevaloración de los jurados con relación a las pruebas periciales, y una subvaloración de su capacidad de sobrevalorar las pruebas testimoniales.

Por lo tanto, el argumento de los autores no es que los jurados sean cognitivamente capaces para valorar las pruebas periciales; más precisamente, 
la idea es que ellos no cometen tantos errores en términos comparativos. Para ellos, la fuerza del escepticismo del common law reside en una comparación implícita entre la sobrevaloración de pruebas periciales y testimoniales; pero esta no parece estar justificada. Jurados (y jueces) sobrevaloran en ambos casos. ¿Por qué entonces adoptar medidas más restrictivas en relación con la primera prueba, y no en relación con la segunda? Como se puede ver, implícita en el argumento de los autores está la constatación de que los jurados cometen errores cognitivos también en el tratamiento de la prueba testimonial. ${ }^{9}$

Sin embargo, incluso antes de discutir las investigaciones que sostienen la tesis sobre la capacidad comparativa de los jurados para valorar pruebas periciales, Schauer y Spellman, en el curso de su razonamiento, mencionan un primer factor que puede haberse escapado a la atención de Vázquez: los jurados frecuentemente aciertan, cuando aciertan, porque siguen señales o indicadores periféricos de autoridad epistémica. Tales indicadores pueden "constituir un fundamento racional para que legos identifiquen expertos y valoren sus juicios" (Schauer y Spellman, 2013, p. 14). En este caso, la calidad epistémica de los indicadores periféricos dependerá de su potencialidad para diagnosticar expertos genuinos.

Los estudios de la psicología social muestran que cuando las personas en general no tienen la experticia necesaria para entender el contenido de una información, a menudo recurren a indicadores periféricos o superficiales para evaluar evidencias y argumentos. ${ }^{10}$ Los seres humanos en general usan dos estrategias para procesar la información comunicada por una persona. La estrategia "periférica" es buscar indicadores para confiar en la persona que nos comunica la información o en la información misma. En cambio, la estrategia "central" de procesamiento de información es trabajar cuidadosamente con la información obtenida. Si se puede hacer una analogía con los sistemas de toma de decisión de Daniel Kahneman, el primer proceso es rápido, automático e inconsciente (Sistema 1); el segundo es lento, con esfuerzo y controlado (Sistema 2) (Kahneman, 2011). En general, las personas demuestran la tendencia a procesar centralmente la informa-

9 Sobre el tema de los errores cognitivos, véase Páez (2019).

${ }^{10}$ Originalmente, véase Petty y Cacioppo (1986). 
ción cuando se sienten motivadas por los recursos cognitivos que tienen o cuando el tema es para ellas relevante.

El recurso a indicadores periféricos independientes del contenido ha sido frecuente por parte de jurados que deciden casos complejos $-i$. e., casos que involucran pruebas científico-tecnológicas o una masa voluminosa de informaciones- (Saks y Spellman, 2016; Gertner, 2018). Entrevistas realizadas con jurados legos indican que ellos acceden más a indicadores superficiales de la validez científica, como credenciales, para valorar la información aportada por el experto. ${ }^{11}$ Estos relatos son consistentes con las indicaciones de las investigaciones experimentales realizadas, que sugieren que "los jurados se involucran en el procesamiento periférico al evaluar los testimonios de especialistas y que las pistas periféricas adquieren un significado adicional a medida que las cuestiones científicas del caso se tornan más complejas" (Sanders, 2003, p. 913).

En un estudio más reciente, Jonathan Koehler et al. identificaron los factores que influyen en las decisiones de jurados legos estadounidenses con respecto a las pruebas que involucran conocimientos ofrecidos por expertos en ciencia forense (Koehler et al, 2017, pp. 401-413). ${ }^{12}$ Los autores testearon la influencia de tres factores: la validación científica del método utilizado por el perito (i. e., si fue probado científicamente, como sugiere el caso Daubert); el background y la experiencia del perito; además de la sofisticación tecnológica del método. La conclusión es que los factores relacionados al background y a la experiencia de los peritos - i. e., indicadores periféricos- ejercen una influencia fuerte y consistente en las decisiones de los jurados. Sin embargo, los factores relativos a la calidad epistémica del método empleado, cuya determinación implicaría algún tipo de ejercicio cognitivo controlado, ejercieron una influencia limitada e inconsistente.

11 Véase Saks y Spellman (2016, cap. 7).

${ }^{12}$ Según los autores, este estudio es resultado de una creciente preocupación de la comunidad científica americana con las condenaciones basadas en ciencias forenses. En 2009, la Academia Nacional de Ciencias de los Estados Unidos publicó un informe crítico cuestionando algunas de las prácticas forenses más comunes, como los análisis de huellas dactilares y de voz y los indicadores de incendios provocados; y, en 2014, el gobierno norteamericano instituyó una Comisión Nacional de Ciencias Forenses con el objetivo de implementar mejoras en las prácticas forenses. 
Lo que los estudios citados anteriormente indican es una tendencia de los jurados a considerar indicadores periféricos en el proceso de valoración de las pruebas periciales. Esto significa que los jurados en realidad no se involucran en un proceso cognitivo de apreciación cuidadosa de las generalizaciones e inferencias utilizadas por los expertos o cualquier otro elemento que requiera algún tipo de procesamiento central, consciente, cuidadoso. Teniendo en cuenta que el concepto de capacidad cognitiva - lo cual es clave en el trabajo de Vázquez- hace referencia a la "memoria de trabajo que dispone un individuo para el procesamiento consciente de información" (Saks y Spellman, 2016, glosario), este no parece manifestarse efectivamente cuando los jurados juzgan pruebas que involucran conocimientos técnico-científicos o una gran cantidad de información. La capacidad cognitiva sería imprescindible, entre otras cosas, para detectar el engaño por parte de expertos y realizar un interrogatorio cruzado, dos de los factores muy importantes para que el juez pueda ejercer una sana crítica con relación a las inferencias de los expertos.

Frente a lo expuesto, sería posible plantear dos objeciones. La primera objeción afirmaría que la propuesta de Vázquez no se enfoca en la (in) capacidad cognitiva de los jurados, sino de los jueces; y que jueces, a diferencia de los jurados, son juzgadores más preparados, generalmente más capaces en términos cognitivos y susceptibles de emplear el tipo de procesamiento central de informaciones que se requiere. Alternativamente, la segunda objeción concedería el argumento anteriormente expuesto, de que los jueces efectivamente no poseen capacidad cognitiva, pero afirmaría entonces que esta sería una razón de peso para apostar en esfuerzos para promover la educación de los magistrados. Intentaré responder cada una de estas objeciones.

Vázquez tiene razón cuando afirma que los estudios sobre las incapacidades de los juzgadores no son concluyentes. En verdad, lo que existe es una falta de estudios psicológicos específicos direccionados a las capacidades de los jueces como juzgadores de los hechos. Numerosos estudios psicológicos sobre las habilidades de los juzgadores en relación con pruebas científicas y técnicas se vienen llevando a cabo hace décadas en los Estados Unidos, pero gran parte se centra en la figura del jurado - el propio artículo de revisión de Schauer y Spellman que Vázquez toma como referencia 
no tiene como foco la capacidad de los jueces-. Dado que las cuestiones de hecho en el sistema norteamericano son frecuentemente juzgadas por el jurado, no hay históricamente mucho interés en investigar las habilidades de los jueces con pruebas de cualquier naturaleza.

Frente a la falta de datos, puede ser plausible presumir que los jueces son más capaces en términos cognitivos que los jurados legos. Después de todo, en el caso de los jueces continentales, ellos fueron aprobados en un proceso de selección competitivo que requirió muchas de sus habilidades intelectuales (interpretaciones, memorias, inferencias); o entonces son personas con reconocida capacidad en virtud de muchos años de experiencia profesional. Por otro lado, los jurados son personas elegidas aleatoriamente en la comunidad. La trayectoria intelectual y la experiencia profesional son factores irrelevantes para la función que deben cumplir. Además de esto, los jueces estarían más capacitados para tratar con pruebas técnico-científicas que los jurados porque no actúan como juzgadores en carácter eventual. Jueces acumulan experiencia a lo largo del tiempo con la repetición del trabajo adjudicativo - ellos serían una especie de "repeat players”- (Damaska, 1997, p. 144). Pero tal presunción puede ser también cuestionada. Para Valerie P. Hans y Michael Saks, una vez que los jurados tienen backgrounds diferenciados, ellos poseen más chances que los jueces de ser cognitivamente capaces para valorar las pruebas periciales (Hans y Saks, 2018). Puede ser que expertos actúen como jurados; y cuantos más jurados figuren en el tribunal, mayores serán las posibilidades de que participen juzgadores con conocimientos especializados. Un jurado que posea formación científica podrá actuar como educador frente a los demás.

Pero el punto central es que los estudios empírico-psicológicos también indican que jurados y jueces no razonan de manera tan diferente. Algunos estudios comparativos indican que los jueces no son menos emotivos que los jurados, por ejemplo; y que el padrón de acuerdo y desacuerdo entre ellos, cuando deciden los casos, no diferencia con la complejidad de las pruebas (Saks y Spellman, 2016, cap. 7). Estas similitudes entre las capacidades cognitivas de los jueces y los jurados nos permiten trazar una analogía importante para la presente discusión. Si los jurados no procesan centralmente las pruebas que involucran conocimientos científicos y técnicos; y si jurados y jueces son semejantes en los aspectos cognitivos 
relevantes; entonces, sí es plausible suponer que los jueces tampoco procesan centralmente las pruebas que involucran conocimientos científicos y técnicos. Esta es una conclusión analógica que obviamente puede ser derogada; sin embargo, no se basa en una mera suposición, sino en datos empíricos y semejanzas relevantes. Creo, por lo tanto, haber respondido la primera objeción.

Pero quizás el punto más importante de la propuesta educacional de Vázquez no es la constatación de que los jueces efectivamente razonan mejor en comparación a los jurados. La cuestión es si los jueces podrían, con ayuda de algunas herramientas procesales, perfeccionar sus capacidades cognitivas de modo de comprender las inferencias presentadas por los peritos. Quiero decir: aun cuando los estudios empíricos indican que los jueces en la actualidad son cognitivamente incompetentes para acceder a las inferencias de los expertos, sería posible educarlos. Si los jueces no tienen la memoria de trabajo necesaria para comprender la información ofrecida por el experto $-\mathrm{y}$ los datos indican que efectivamente no la parecen tener-, la información no será procesada centralmente y de modo consciente. La idea es que los jueces podrían adquirir (en términos potenciales) dicha memoria de trabajo. Cuando la cuestión es reformulada así, los datos empírico-psicológicos que se deben examinar son los relacionados con la adquisición y manutención de la experticia.

¿Entonces, bastaría educarlos? La respuesta me parece ser no, o al menos no sin que esto no implique costos muy altos y con resultados todavía inciertos para la administración de la justicia. ${ }^{13}$ Si nos tomamos en serio la tarea de educar a los jueces, la literatura que importaría analizar sería aquella sobre adquisición de experticia y otras buenas performances. Pero antes de todo, sería necesario definir el nivel de performance deseado de los jueces para que comprendan y controlen las inferencias de los expertos. ${ }^{14}$ Para adquirir la experticia, los estudios muestran que es necesario la manutención de experiencias y prácticas específicas. Son exigidas muchas

${ }^{13}$ De todos modos, creo que estamos de acuerdo con que los jueces tienen el potencial de desarrollar las capacidades necesarias para comprender las inferencias de los expertos. La cuestión es si el tiempo y el esfuerzo involucrados hacen que la propuesta sea factible.

${ }^{14}$ Michele Chi diseña una escala de competencias que van desde el principiante al máster, aquel que está de hecho por encima del experto (Chi, 2006, p. 21). 
horas de entrenamiento para desarrollar un conjunto de actividades llamadas de deliberate practice (Ericsson y Pool, 2017, pp. 97-100). ${ }^{15}$ Ejecutar repetidamente una tarea en la rutina sin intención de mejorar y sin recibir un feedback no contribuye para la adquisición de una experticia. Una práctica similar, pero diferente, y que constituye una etapa para alcanzar la experticia, es la llamada purposeful practice (Ericsson, 2006, pp. 14-17). Pero esta también exige que uno tenga bien definido el foco, salga de su zona de confort y reciba feedback. ¿Qué tipo de práctica sería necesaria a los jueces para lograr la performance que los modelos educacionales les piden? Si tomamos como referencia la literatura sobre adquisición y manutención de la experticia y niveles similares de performance, aunque sea permitido suponer que los jueces pueden recibir feedback con relación a sus decisiones de (in)admisibilidad de pruebas periciales — pues las instancias superiores suelen tratar el juicio de admisibilidad como una cuestión de derecho sujeta a revisión judicial-, es cuestionable la idea de feedback institucional con relación a sus decisiones de valoración de las inferencias de los expertos.

Ya no puede seguir explorando la literatura referida sin comprometer el espacio que me fue permitido en esta discusión. Así que termino esta sección con una cita de Saks y Spellman y que me permite introducir el punto siguiente:

Los humanos no venimos bien equipados para evaluar y usar evidencia científica y estadística. Sin entrenamiento especial, y a menudo incluso con dicho entrenamiento, no lo hacemos bien. Por otro lado, lamentablemente somos bastante buenos en aceptar una conclusión afirmada por alguien que creemos que es una autoridad en un tema. Quizás estos son problemas que no se pueden resolver sin cambios radicales - y quizás inaceptables - en nuestro sistema (Saks y Spellman, 2016, capítulo 7, traducción e itálicos por mi cuenta).

Las consideraciones realizadas me permiten conducir las cuestiones anteriores al punto final de mi discusión: el presupuesto teórico-episté-

${ }^{15}$ Véase, adicionalmente (Ericsson, Charness, Feltovich y Hoffman, 2006, capítulos de la parte IV). 
mico del modelo educacional de Allen y Vázquez — esto es, la idea de que la deferencia es siempre irracional y, por lo tanto, contraria a una de las aspiraciones centrales de nuestros sistemas jurídicos-.

\section{El presupuesto de carácter teórico-epistémico}

El argumento de Vázquez tiene también un componente teórico-epistémico. La autora presupone que la deferencia es siempre una actitud irracional. Esta posición implica cierta concepción de racionalidad que no me parece adecuada para dar cuenta de lo que sucede en el contexto judicial. Hay muchas maneras de tomar decisiones racionales; y algunas de ellas no exigen la comprensión del contenido de la decisión. En este apartado voy a defender que un modelo crítico de deferencia epistémica por parte de jueces puede ser no solo racional, sino también coherente con uno de los elementos distintivos de la toma de decisiones en el contexto judicial, a saber, el concepto de "autoridad".

A primera vista, Vázquez puede tener razón acerca de la irracionalidad de la deferencia a la autoridad. Tradicionalmente, la lógica del recurso a la autoridad ha sido realmente problemática. ¿Por qué una persona debe abandonar su autonomía intelectual, el elemento que la distingue como agente racional, para confiar en las palabras del otro? No es sorprendente que el argumento de autoridad — sea epistémica, sea política- haya sido históricamente considerado una falacia por los estudiosos de la lógica. Fue precisamente John Locke quien, en 1690, llamó a este tipo de argumento argumentum ad verecundiam, que literalmente significa "modestia" al cuestionar las palabras de una autoridad. Para Locke, el argumentum ad verecundiam fue visto como una forma de coerción. Sin embargo, este es un entendimiento lógico obsoleto. Los libros de lógica informal que usamos hoy tratan el argumento de la autoridad como un tipo de inferencia frecuente y obviamente derrotable, pero que todavía puede ser legítimo. Su fuerza dependerá de la satisfacción de algunas condiciones (Walton, 1997; Govier, 1985). Estas condiciones funcionan como preguntas críticas que uno que apela a la autoridad debe contestar, y se refieren a una serie de situaciones que ponen en duda el pronunciamiento de la auto- 
ridad experta. Son factores no solo epistemológicos (como la pregunta “¿qué dice el experto que es consistente con lo que afirman otros en la misma área?"), sino también éticos (como la pregunta “¿es el experto personalmente confiable?”. ${ }^{16}$ Efectivamente, las preguntas críticas funcionan como los indicadores periféricos de la buena experticia de que hablaba en el apartado anterior.

Susan Haack, en su reciente artículo publicado en Quaestio Facti, ofrece una serie de preguntas (y respuestas) que nos ayudan a operacionalizar mejor los indicadores periféricos de la buena experticia (Haack, 2019). Tales preguntas funcionan como consejos prácticos que la autora presenta para ayudar al juzgador lego a evaluar la fiabilidad de la prueba pericial. Por ejemplo, con relación al indicador "publicación en revista científica revisada por pares", uno de los factores de fiabilidad de la prueba pericial estipulados en el caso Daubert, Haack sugiere las siguientes preguntas: cuando el artículo fue revisado por pares, ¿se le pidió al autor que él mismo indicara los árbitros? ¿Cuántos árbitros fueron consultados? ¿Cuál es el estatus o la clasificación de la revista? ¿Cómo se sustenta financieramente la revista? ¿Ha habido alguna forma de retractación o expresión de preocupación por parte del editor? ¿El trabajo ya recibió muchas citas? Es obvio que tales preguntas apuntan a señales que pueden eventualmente fallar. Lo que importa es que efectivamente exista una correlación entre los indicadores y la fiabilidad de la prueba pericial, y que los juzgadores legos - jurados o jueces - no queden cognitivamente perdidos para ponerlos en funcionamiento (Haack, 2019, p. 19). "La idea, si se prefiere decir así, es proporcionar los medios para gradualmente dar un pequeño empujón a los actores jurídicos en dirección a la identificación de mejores expertos y el escrutinio mejor informado del testimonio del experto", ${ }^{17}$ concluye Haack (2019, p. 28).

De todo lo anterior, está claro que una de las preocupaciones que deben tenerse en cuenta al formular dichos indicadores periféricos o superficiales

16 Son conocidos los esquemas de preguntas diseñados por Walton, Reed y Macagno (2008).

${ }^{17}$ El concepto de empujón (nudge) en las ciencias comportamentales contrasta justamente con la idea de que vale la pena invertir en modelos de educación. Es un tipo de intervención no costosa en el ambiente institucional de toma de decisiones que parte del presupuesto de que el razonamiento de los seres humanos es frecuentemente falible. Ver Thaler y Sunstein (2008). 
es el requisito de que no se refieren a las razones que solo el experto o su comunidad de expertos pueden acceder. Además, tales indicadores pueden llevar a los jueces a tomar decisiones incorrectas eventualmente; pero seguir una lista de señales que actúan como buenas razones para confiar en el experto, si están bien diseñadas, permitirá tomar más decisiones correctas que incorrectas in the long run.

La idea de buscar buenas razones para confiar en los expertos es representativa del trabajo de John Hardwig. En un conjunto de artículos sobre la autoridad de los expertos, el epistemólogo estadounidense desarrolló una estructura útil para explicar cómo se sucede el apelo racional y la confianza en los expertos. Esta estructura nos permite distinguir dos tipos de razones que están en juego, y nos puede ayudar a entender cuáles son las razones que los jueces pueden y deben considerar para decidir sobre el valor de las informaciones ofrecidas por los expertos. La estructura de Hardwig para explicar la deferencia racional a los expertos es la siguiente:

Si $A$ [el lego, el juez] tiene buenas razones para creer que $B$ [el experto] tiene buenas razones para creer que $p$ [la información probatoria], entonces $A$ tiene buenas razones para creer que $p \cdot{ }^{18}$

La estructura indica que no se trata de una relación entre personas iguales y cuyas razones tienen igual valor epistémico - el experto sabe más, y sus razones para creer en una hipótesis probatoria son diferentes-. Pero el juez tiene como y debe ser estimulado a considerar críticamente las razones que tiene para creer que el experto tiene buenas razones. $\mathrm{Y}$ estas razones, que podemos llamar de "razones de segundo orden", funcionan, en la práctica, como los indicadores periféricos.

Un punto que merece destacarse es que el empleo de indicadores periféricos presupone la existencia de un ambiente deliberativo. Esto significa que la deferencia al experto no es ciega, sino crítica. Luego, si, por un lado, es apropiado decir (en el sentido expuesto por Joseph Raz) ${ }^{19}$ que las razones que justifican apelar a la autoridad epistémica del experto son independien-

\footnotetext{
${ }^{18}$ Esta fórmula aparece por la primera vez en Hardwig (1985, p. 336, la traducción es mía).

${ }^{19}$ Sobre razones de autoridad, $\operatorname{Raz}(1990)$.
} 
tes del contenido del que la autoridad afirma y excluyen la deliberación o cualquier forma de ponderación de razones de primer orden — aquellas que solo poseen los experto y sus pares-, por otro lado, no sucede lo mismo en relación con las razones de segundo orden que remiten a los indicadores periféricos que pueden tornar la deferencia racional. ${ }^{20}$ Tales razones de segundo orden continúan siendo relativamente independientes del contenido de lo que el experto afirma (Herdy, 2019, pp. 23-46), pero con respecto a ellos no podemos suponer que hay una especie de deferencia ciega o la completa ausencia de deliberación racional. Muy por el contrario: la toma de decisión con base en el balance de los indicadores periféricos requiere herramientas institucionales que proporcionen un ambiente saludable para la discusión crítica.

En esta línea de razonamiento, también es posible decir que un modelo educacional para el tratamiento de la prueba pericial propuesto por Vázquez es menos democrático en comparación con un modelo deferencial crítico que he bosquejado precedentemente - algo que suena sorprendente- . Un modelo educacional se ocupa del problema de la justificación de la decisión judicial a partir de la perspectiva de los jueces o jurados esto es, a partir del ejercicio cognitivo de los juzgadores de los hechos-. Sin embargo, un modelo deferencial crítico ofrece una justificación a un mayor número de personas. No sería solo el juez científicamente educado quien tendría la capacidad de comprender las buenas razones para creer lo que los expertos dicen (o decidir en qué experto confiar cuando hay un conflicto), sino todos los participantes de la sociedad en cuestión - esto es, los destinatarios finales de una administración racional de la justicia-.

Lo que acabo de decir cuestiona el supuesto teórico-epistémico de que la deferencia es siempre irracional. Pero es necesario decir más: la actitud de deferencia (crítica) que se propone es característica de la justificación judicial y, por lo tanto, coherente con las aspiraciones centrales de nuestros sistemas jurídicos. La deferencia a los expertos no difiere del tipo de deferencia que ocurre cuando los jueces deciden aplicar una regla o precedente

${ }^{20}$ Comparto una concepción débil de independencia del contenido y deliberación, en que factores sustantivos, como la posición de la autoridad, son importantes y deben ser considerados para la determinación de la razón para la acción (o decisión). Véase Gur (2011). 
contrario a sus creencias morales o políticas. La suposición de que la deferencia violaría la "idea normal" del proceso de decisión judicial ignora gran parte de lo que sucede en los tribunales, y el elemento que parece distinguir el razonamiento jurídico -el carácter autoritario de las decisiones (Raz, 1990) - . Los argumentos de autoridad y la consecuente deferencia del juez son fenómenos que invaden los tribunales: se producen toda vez que aplicamos reglas o precedentes como razones excluyentes en relación con las consideraciones de primer orden que podamos tener. Ahora, la deferencia a los hechos de carácter técnico o científico no parece ser un fenómeno tan diferente. Efectivamente, es un fenómeno que revela la deferencia del juez de manera muy similar a la que ocurre cuando las reglas y los precedentes se aplican como fuentes de autoridad. ${ }^{21}$

Además, podemos decir que la deferencia hacia el experto podría clasificarse como una deferencia aún más liviana o tenue en comparación con la deferencia de los jueces a las fuentes de autoridad normativa. La autoridad de una premisa normativa de la inferencia judicial es más sentida, más fuerte, porque en este caso el juez es cognitivamente capaz de formar una opinión sobre el tema. Pensemos en una decisión sobre la posibilidad de la eutanasia o del aborto. El juez es un experto en cuestiones normativas; sin embargo, en muchos casos opta por deferir, por razones de segundo orden (que contrarían su íntima convicción), al que estableció anteriormente el legislador o el juez/tribunal. ¿Por qué el caso de deferir al conocimiento experto sería tan diferente? Si incluso con respecto a la norma admitimos y eventualmente estimulamos la deferencia del juez (pensemos en los modelos formalistas de toma de decisión), ¿por qué no admitir la posibilidad de modelos deferenciales con respecto a los hechos? ${ }^{22}$

\footnotetext{
${ }^{21}$ Sobre el tema, véase Herdy (2014).

${ }^{22}$ Además, la apuesta en un modelo deferencial crítico es coherente también con la caracterización de la actitud proposicional de los jueces frente a los hechos como de aceptación (Ferrer Beltrán, 2005). Un modelo educacional supone que los jueces tengan la capacidad de formar creencias sobre las proposiciones de hecho afirmadas por los expertos.
} 


\section{Conclusión}

En 1878, Charles S. Peirce declaró que "si las creencias apaciguan las mismas dudas produciendo las mismas reglas de acción, entonces las simples diferencias en la forma en que somos conscientes de ellas no pueden hacerlas creencias diferentes". Es posible que estemos a "tocar una melodía en diferentes escalas", pero eso "no es lo mismo que tocar diferentes melodías" (Peirce, 1986, p. 264). Tal vez realmente estoy tocando la misma melodía que Vázquez, pero en otra escala. Como dije en el inicio, sus propuestas concretas para un nuevo diseño de las pruebas periciales en nuestros sistemas jurídicos me parecen adecuadas y oportunas. Los puntos que recibieron mi atención en esta discusión con la autora son relativos a sus presupuestos empíricos y teóricos. Identifiqué y discutí contra los dos presupuestos que justifican la opción de Vázquez por un modelo educacional para la valoración judicial de la prueba pericial. El primer presupuesto es empírico-psicológico, y sustenta que los déficits de los jueces no son cognitivos; el segundo presupuesto es teórico-epistémico, y sustenta que la deferencia es siempre irracional.

Precisamente en vista de la evidencia empírica disponible sobre las capacidades y la actuación de los jurados y jueces, creo que una forma de deferencia crítica del juez al experto parece ser la mejor opción. Como he argumentado, la deferencia no necesita ser ciega, ya que podemos pensar en indicadores periféricos racionales. Cuando se aplican de manera consistente, tales indicadores no solo garantizarían la deferencia crítica hacia los epistémicamente capaces de decidir con racionalidad, sino que también aumentan el valor democrático de la decisión judicial. Además, la idea de que un juez debe aceptar las palabras de otros sin tener que acceder a sus méritos es una característica clave del razonamiento judicial.

Es cierto que el problema de la abdicación judicial del juez en relación con los hechos del litigio que demandan conocimientos expertos puede ser más bien visto como una instancia de un problema institucional mayor. Este problema consiste en compatibilizar uno de los principales ideales de las democracias liberales, que garantiza a los individuos el derecho de participar directamente y/o controlar racionalmente las decisiones estatales que les conciernen, con la posición epistémica de desigualdad en que se encuentran 
los legos frente a los expertos en relación a una serie de temas que son objeto de decisión en la esfera pública. ${ }^{23}$ El problema se presenta como un desafío a la propia gobernanza democrática en sociedades que aspiran por políticas públicas basadas en evidencia. Cuando se aspira por decisiones fundamentadas en los avances científicos y tecnológicos, ni todos pueden hablar y ni todas las charlas tienen igual valor. El modelo deferencial crítico cuyas líneas generales he bosquejado incorpora esas tensiones e intenta ofrecer una solución que, en mi opinión, es tanto racional cuanto democrática.

\section{Bibliografía}

Allen, R. (2013). The Conceptual Challenge of Expert Evidence. Revista Discusiones Filosóficas, 14(23), 41-65.

Chi, M. T. H. (2006). Two Approaches to the Study of Expert's Characteristics. En K. A. Ericsson, N. Charness, P. Feltovich, y R. R. Hoffman, (eds.), Cambridge handbook of expertise and expert performance (pp. 21-30). Cambridge, UK: Cambridge University Press. Damaska, M. (1997). Evidence Law Adrift. New Haven: Yale University Press.

Dwyer, D. (2008). Judicial Assessment of Expert Evidence, Cambridge: Cambridge University Press.

Edmond, G. (2012). Advice for the Courts? Sufficiently Reliable Assistance with Forensic Science and Medicine (Part 2). The International Journal of Evidence \& Proof, 16, 263-297.

Edmond, G. y Roberts, A. (2011). Procedural Fairness, the Criminal Trial and Forensic Science and Medicine. Sydney Law Review, 33, 359-394.

Epstein, R. (1993). Judicial Control over Expert Testimony: Of Deference and Education. Northwestern University Law Review, 87(4), 1156-1165.

Ericsson, K. A. (2006). An Introduction to The Cambridge Handbook of Expertise and Expert Performance: Its Development, Organization, and Content. En K. A. Ericsson, N. Charness, P. Feltovich, y R. R. Hoffman, (eds.), Cambridge handbook of expertise and expert performance (pp. 3-20). Cambridge, UK: Cambridge University Press. 
Ericsson, A. y Pool, R. (2017). Peak: Secrets From the New Science of Expertise. Boston: Mariner Books.

Faigman, D. (2014). Group to Individual (G2i) Inference in Scientific Expert Testimony. The University of Chicago Law Review, 81(2), 417-480.

Ferrer Beltrán, J. (2005). Prueba y verdad en el derecho, Madrid: Marcial Pons. Gascón, M. (2016). Conocimientos expertos y deferencia del juez. DOXA, (39), 347-365.

Gertner, N. y Sanders, J. (2018). Alternatives to Traditional Adversary Methods of Presenting Scientific Expertise in the Legal System. Daedalus, 147(4), 135-151.

Govier, T. (1985). A Practical Study of Argument. Belmont CA: Wadsworth. Gur, N. (2011). Are Legal Rules Content-Independent Reasons?. Problema: Anuario de Filosofía y Teoría del Derecho, 5, 175-210.

Haack, S. (2019). Judging Expert Testimony: From Verbal Formalism to Practical Advice. Quaestio facti, 1, 13-30.

Hans, V. P. y Saks, M. J. (2018). Improving Jury \& Judge Evaluation of Scientific Evidence. Daedalus, 147(4), 164-180.

Hardwig, J. (1985). Epistemic Dependence. The Journal of Philosophy, 82(7), 335-349.

Herdy, R. (2014). Dependencia epistémica, antiindividualismo y autoridad en el derecho. Isonomía, 40, 119-146.

Herdy, R. (2019). Appeals to Expert Opinion in High Courts. En M. Nogueira de Brito, R. Herdy, G. Damele, P. Moniz Lopes, y J. Silva Sampaio (eds.), The Role of Legal Argumentation and Human Dignity in Constitutional Courts (pp. 23-46). Stuttgart, Franz Steiner Verlag, 2019. Imwinkelried, E. J. (1997). The Next Step in Conceptualizing the Presentation of Expert Evidence as Education: The Case for Didactic Trial Procedures. The International Journal of Evidence and Proof, 1(2), 128-148.

Kahneman, D. (2011). Thinking, Fast and Slow. New York: Farrar, Straus and Giroux.

Koehler, J. J., Schweitzer, N. J., Saks, M. J., y McQuiston, D. E. (2016). Science, Technology, or the Expert Witness: What Influences Jurors' 
Judgments About Forensic Science Testimony? Psychology, Public Policy, and Law, 22, 401-413.

Lempert, R. (1981). Civil Juries and Complex Cases: Let's Not Rush to Judgment. Michigan Law Review, 80(1), 68-132.

Miller, J. S. y Allen, R. (1993). The Common Law Theory of Experts: Deference or Education? Northwestern University Law Review, 87(4), 1131-1147.

Moore, A. (2017). Critical Elitism: Deliberation, Democracy, and the Problem of Expertise. Cambridge: Cambridge University Press.

Páez, A. (2019). Los sesgos cognitivos y la legitimidad racional de las decisiones judiciales, disponible en https://philpapers.org/rec/PEZLSC. Peirce, C. S. (1986). Writings of Charles Sanders Peirce. En Kloesel, C. J. (ed.), vol. 3, Bloomington: Indiana University Press.

Petty, R. y Cacioppo, J. T. (1986). Communication and Persuasion: Central and Peripheral Routes to Attitude Change. New York: Springer-Verlag. Raz, J. (1990). Authority and Justification. En J. Raz (ed.) Authority (pp. 115-141). New York: New York University Press.

Saks, M. y Spellman, B. (2016). The Psychological Foundations of Evidence Law. New York: NYU Press.

Sanders, J. (2003). The Merits of Paternalistic Justification. Seton Hall Law Review, 33, 881-941.

Schauer, F. y Spellman, B. (2013). Is Expert Evidence Really Different? Notre Dame Law Review, 89(1), 1-26.

Thaler, R. A. y Sunstein, C. (2008). Nudge: Improving Decisions about Health, Wealth, and Happiness. New York: Penguin Books.

Vázquez, C. (2015). De la prueba científica a la prueba pericial. MadridBarcelona: Marcial Pons.

Walton, D. (1997). Appeal to Expert Opinion: Arguments from Authority. Pennsylvania: Pennsylvania State University Press.

Walton, D., Reed, C. y Macagno, F. (2008). Argumentative Schemes. Cambridge: Cambridge University Press. 